\title{
SOME PROPERTIES OF SPHERICAL CURVES, WITH APPLICATIONS TO THE GYROSCOPE*
}

In his paper entitled On the gyroscopet, Professor Osgood has reduced to the utmost simplicity certain important aspects of the theory of the motion of a rigid body which is dynamically symmetric about an axis through the center of mass. The present study derives its inspiration from his article. A fundamental rôle is there played by the geodesic curvature of the trace on the unit sphere with center at the fixed point, or at the center of mass, of the point where this sphere is cut by the axis of symmetry, and this fact suggests that it might be desirable to have on hand more information about the intrinsic properties of spherical curves.

Accordingly, the first part of the present paper is devoted to the development of a number of such properties. The subject, however, is not without interest for its own sake, and suggests numerous extensions and related problems; for instance, a systematic study of the relationships of certain properties of the curvature as a function of the length of arc with the corresponding geometric properties of the curve. One such property which has already received a good deal of attention is the "four vertex theorem" (Vierscheitelsatz) for plane ovalsł. Among other questions that might be raised are such simple ones as the following: what functional character of the curvature, in addition to periodicity, insures a closed curve on the sphere? When is a plane, or spherical curve, asymptotic to some closed curve? What interesting comparison theorems are there for pairs of curves whose curvatures stand in simple relationships of equality or inequality? And there is the further question to be considered of curves on more general surfaces.

The second part of the paper makes application of the results of the first part to the theory of the gyroscope. While some apparently new facts are there brought to light, mention should be made of a new way of establishing

\footnotetext{
* Presented to the Society, April 28, 1923.

†These Transactions, vol. 23 (1922), pp. 240-264. This paper will be referred to hereafter by the initial 0 .

\$See, for instance, Blaschke, Vorlesungen über Differentialgeometrie, vol. I, 1921, p. 16.
} 
some classical results. It will be recalled* that certain theorems on the sense of precession of the gyroscope, and inequalities on the longitudinal motion of the spherical pendulum, have hitherto required the use of the theory of elliptic functions, or of Cauchy's integral theorem, for their establishment, while others depend simply on the appraisal (Abschätzung) of definite integrals. It turns out that exactly those results which have previously required the less elementary methods are simple consequences of our present geometric theorems.

In addition to the applications mentioned, there will be found in the second part of the paper certain further results and formulas which may well be of use in connection with problems on the gyroscope.

\section{PART I. ON CERTAIN INTRINSIC PROPERTIES OF CURVES}

1. Plane curves whose curvatures approach limits as the arc lengths increase indefinitely. The need of care in the study of qualitative properties of curves is illustrated by a loose statement in the authorized German translation of Cesàro's excellent book on intrinsic geometry.t In the discussion of plane curves the following sentences appear, in which $\varrho$ is the radius of curvature, and $\varphi$ the angle between a fixed line and the tangent at the point corresponding to the arc length $s$ : "Nur wenn $s$ zusammen mit $q$ unbegrenzt zunimmt, kann es vorkommen, da $\varrho$ sich einem von Null verschiedenen Grenzwerte $a$ nähert. Alsdann windet sich die Kurve, anstatt sich um einen Punkt herumzuwickeln, asymptotisch um einen Kreis vom Radius a, und zwar innerhalb oder außerhalb desselben, je nachdem der absolute Betrag von e sich oberhalb oder unterhalb seines Grenzwertes hält." The following example shows that from the knowledge that $\rho$ approaches a limit as $s$ becomes infinite we cannot infer that an asymptotic circle exists.

Let $s_{n}$ denote the sum of the first $n$ terms of the harmonic series, $s_{n}=1$ $+1 / 2+1 / 3+\cdots+1 / n$. We describe about each point $\left(s_{n}, 0\right)$ of the $x, y$-plane, a circle of radius $a$. We then erase the upper halves of these circles, and unite the lower halves to a single continuous curve, with continuous curvature, by joining the two most distant extremities of each pair of successive semicircles by an arch of an ellipse with transverse axis along the $x$-axis, so chosen as to make the curvature continous. Thus, the first arch has its major axis terminating in the points $(1-a, 0)$ and $(3 / 2+a, 0)$, the second, in $(3 / 2-a, 0)$ and $(11 / 6+a, 0)$, and so on.

\footnotetext{
* See 0, p. 260, end of page, and Appell, Traité de Mécanique Rationnelle, vol. I, Paris, 1902, p. 501.

† Vorlesungen über natïrliche Geometrie, Leipzig, 1901, p. 12.
} 
Here the curvature is seen to approach the limit $1 / a$, but no asymptotic circle exists. The example can evidently be modified to bring out a number of different facts. For instance, the initial set of circles may be taken with their centers on a closed curve, say a large circle. We shall then have a bounded curve exhibiting the same lack of an asymptotic circle.

A statement that can be made, however, is the following:

'I'HEOREM I. If a plane curve has curvature, $K$, which is a continuous function of the arc length, $s$, and if, as $s$ becomes infinite, $K$ approaches a limit different from 0 while always increasing or always decreasing, then the curve has an asymptotic circle, approached from without, or within, respectively.

As the proof of this theorem is analogous to that of a later one on spherical curves (p. 509), it will not be given. Instead, we shall prove a theorem which is essentially broader in its hypothesis, and whose analogue for spherical curves is neither so simple of treatment, nor so interesting from the point of view of dynamics:

'THEOREM II. If there exist two constants, $\alpha>0$, and $s_{0}$, such that for $s_{0}<s<\infty, K(s)>\alpha$, and if $K(s)$ is of bounded variation, then the curve defined by $K=K(s)$ has an asymptotic circle.

A consideration of the total variation, $t(s)$, of $K(s)$, makes it obvious that $K(s)$ must approach a limit, $k$. Moreover, if $\tau(s)$ is the angle which the tangent to $\mathfrak{E}$, the curve under consideration, makes with a fixed direction, then $K$ is also of bounded variation when considered a function of $\tau$ $\left(\tau(s)=\int_{s_{0}}^{s} K(s) d s\right)$. Now the coordinates, $x(s), y(s)$, of $P$, on $(5$, referred to appropriate axes, are given by

$$
x=\int_{0}^{\tau} \frac{\cos \tau}{K(\tau)} d \tau, \quad y=\int_{0}^{\tau} \frac{\sin \tau}{K(\tau)} d \tau .
$$

From these formulas it is not difficult to show that the successive maxima and minima of $x$ and $y$ approach limits, and that the mean of these limits for $x$, and the mean for $y$, give the coordinates of the center of an asymptotic circle.

The maxima, $x_{n}^{\prime \prime}$, of $x$ occur for $\tau=\left(2 n+\frac{1}{8}\right) \pi$, and the minima, $x_{n}^{\prime}$, for $\tau=\left(2 n+\frac{8}{2}\right) \pi$. If the interval of integration in the expression for $x_{n}^{\prime \prime}$.be subdivided at these points, we may use the law of the mean in each sub-interval, and write 


$$
\begin{aligned}
x_{n}^{\prime \prime}= & \int_{0}^{\left(2 n+\frac{1}{3}\right) \pi} \frac{\cos \tau}{K(\tau)} d \tau=\int_{0}^{\frac{\pi}{2}} \frac{\cos \tau}{K(\tau)} d \tau+\sum_{i=0}^{i=2 n-1} \int_{\left(i+\frac{1}{2}\right) \pi}^{\left(i+\frac{8}{2}\right) \pi} \frac{\cos \tau}{K(\tau)} d \tau \\
= & \frac{1}{K\left(\tau_{0}\right)}-\frac{2}{K\left(\tau_{1}\right)}+\frac{2}{K\left(\tau_{2}\right)}+\cdots+\frac{2}{K\left(\tau_{2 n}\right)} \\
= & \frac{1}{K\left(\tau_{0}\right)}-2\left\{\left[1 / K\left(\tau_{1}\right)-1 / K\left(\tau_{2}\right)\right]+\left[1 / K\left(\tau_{3}\right)-1 / K\left(\tau_{4}\right)\right]\right. \\
& \left.+\cdots+\left[1 / K\left(\tau_{2 n-1}\right)-1 / K\left(\tau_{2 n}\right)\right]\right\},
\end{aligned}
$$

where $\tau_{0}, \tau_{1}, \ldots, \tau_{2 n}$ are appropriate mean values of $\tau$.

Now, since $K(\tau)>\alpha>0,1 / K(\tau)$ is of bounded variation with $K(\tau)$, for $|1 / K(b)-1 / K(a)|<|K(b)-K(a)| / \alpha^{8}$. Hence the terms in the braces are the first $n$ terms of an absolutely convergent series. Accordingly, $x_{n}^{\prime \prime}$ approaches a limit, $x^{\prime \prime}$. Similarly, $x_{n}^{\prime}, y_{n}^{\prime \prime}$ and $y_{n}^{\prime}$ approach limits, $x^{\prime}, y^{\prime \prime}$, and $y^{\prime}$. Let us denote by $a$ and $b$ the means, $\left(x^{\prime}+x^{\prime \prime}\right) / 2$ and $\left(y^{\prime}+y^{\prime \prime}\right) / 2$, of these limits, and by $r$, the limit, $1 / k$, of $1 / K(\tau)$. Then, since $\int \frac{\cos \tau}{K(\tau)} d \tau$ and $\int \frac{\sin \tau}{K(\tau)} d \tau$, taken over any interval whose end points correspond to successive integral multiples of $\pi / 2$, approach $r$, or $-r$, according to the interval selected, we see that the points of $\mathfrak{E}$ of maximum abscissas, maximum ordinates, minimum abscissas, and minimum ordinates, approach $(a+r, b),(a, b+r),(a-r, b)$ and $(a, b-r)$, respectively. Let $\tau_{1}$ be a number, corresponding to a given $\varepsilon>0$, such that for $\tau>\tau_{1}$, the differences between these four variables and their limits are numerically less than $\varepsilon$, and also that $|1 / K(\tau)-1 / k|<\varepsilon$. This means that for $\tau>\tau_{1}$, the extremes of the coordinates of $\mathfrak{C}$ differ by less than $\varepsilon$ from the corresponding extremes for the circle $C: X=a+r \sin \tau$, $Y=b-r \cos \tau$. From this we infer that for any point of $\mathbb{E}$ for $\tau>\tau_{1}$,

$$
|x(\tau)-X(\tau)|<\varepsilon+\int|[1 / K(\tau)-1 / k] \cos \tau| d \tau<2 \varepsilon,
$$

where the interval of integration is from the greatest multiple of $\pi / 2$ less than $\tau$, to $\tau$. A similar inequality will hold for $y(\tau)-Y(\tau)$. Thus $\mathbb{c}$ ultimately lies entirely in any region containing the circumference $C$ in its interior; that is, $C$ approaches $C$ in the weak sense. It is easily shown, however, that the approach has also the stronger sense, namely, that the difference of the direction angles, $\tau$ and $\boldsymbol{t}^{\prime}$ of $\mathbb{C}$ and $C$, at two points, one on each curve, also 
approaches 0 has $\tau$ increases indefinitely and the two points approach coincidence. This is because of the constant curvature of $C$, which has as consequence the finite distance apart of any pair of points at which the directions of $C$ differ by a finite amount. The required inequalities may readily be supplied, and the proof of the theorem thus completed.

2. Spherical curves and spherical evolutes. The most convenient analytical tool for the present study appears to be the vector. We shall denote vectors by Clarendon letters, $a, b, P$, etc., and employ the Gibbs notation, $\boldsymbol{a} \cdot \boldsymbol{b}$ for the scalar product, $a \times b$ for the vector product, and $(a, b, c)=a \cdot(b \times c)$ for the triple product. The vector algebra required goes little beyond the distributive laws, and the expansion formula $a \times(b \times c)=(a \cdot c) b-(a \cdot b) c$. Primes will be used for one purpose only, namely, to denote derivatives with respect to the arc-length, $s$, of $\mathbb{C}$. Unit vectors along the coordinate axes will be denoted by $i, j, k$, and their senses we shall assume fixed once for all, and so related to the definition of vector product that $(i, j, k)=+1$. The magnitude of a vector will be denoted by the corresponding italic letter.

Let the curve $\mathbb{S}$ lie on the unit sphere, $S$, and let a variable point of $\mathbb{C}$ be characterized by the unit position vector, $P=x i+y j+z k$, with origin at $O$, the center of $S$. We shall restrict ourselves to curves such that $x, y$ and $z$ have continuous derivatives with respect to $s$ of the first three, and, in some cases, the first four orders. From $\boldsymbol{P}$ are derived the tangent vector

$$
T=P^{\prime}
$$

and the curvature vector

$$
K=T^{\prime}=P^{\prime \prime}
$$

For these we have the relations

$$
P^{\mathbf{y}}=1
$$

since $\mathbb{C}$ is on the unit sphere, and

$$
T^{z}=1
$$

since $\left(P^{\prime}\right)^{2}=\left(d s^{\prime} d s\right)^{2}=1$.

Furthermore, by differentiating these relations, we find

$$
\begin{aligned}
& P \cdot T=0, \\
& T \cdot K=0,
\end{aligned}
$$


and by further differentiation,

$$
\begin{aligned}
& P \cdot K=-T^{2}=-1, \\
& P \cdot K^{\prime}=-T \cdot K=0 \\
& T \cdot K^{\prime}=-K^{2} .
\end{aligned}
$$

The unit principal normal vector, $N$, of $\mathbb{S}$ will coincide in direction with $K$ We shall give it the same sense, so that

$$
N=K / K=\varrho K
$$

where $K$ and $\varrho$ are the curvature and radius of curvature of $(5$. Formula (7) shows that $K$ always makes an obtuse angle with the position vector, and that its magnitude, the curvature, is never less than 1.

The unit binormal vector is defined as

$$
B=T \times N
$$

If the initial point of $B$ be placed at $O$, its tip will mark a point, $Q$, on $S$ which is the spherical center of curvature of $\mathbb{E}$ for the point $P$ (the tip of $P$ ). We define the quantity $R, 0 \leqq R \leqq \pi$, by the equations

$$
\sin R=\varrho, \quad \cos R=P \cdot B
$$

and call it the spherical radius of curvature. The same term will be used occasionally for the great circle arc, $Q P$, of which it is the length. If, with $Q$ as center, and with spherical radius $R$, a circle be described on the sphere, this will be the osculating circle of $\mathbb{E}$ at $P$.

As $P$ moves along $\mathbb{C}, Q$ will, in general, trace a curve, $\mathfrak{F}$, the spherical evolute of $\mathfrak{c}$. The point diametrically opposite $Q$ will trace a symmetric curve, similarly related to $(5$. But the particular evolute here defined is selected by the sense given to the binormal vector. A reversal of the sense of increasing $s$ on $\sqrt{ }$ would interchange the evolutes.

The Frenet formulas take the form

$$
T^{\prime}=\frac{N}{\varrho}, \quad N^{\prime}=\frac{B}{\tau}-\frac{T}{\varrho}, \quad B^{\prime}=-\frac{N}{\tau},
$$


where $1 / \tau$ is the torsion of $\mathfrak{C}$. From the third Frenet formula, and equations (12), (10) and (7), we see that the torsion is the negative of the derivative with respect to $s$ of the spherical radius of curvature,

$$
R^{\prime}=-\frac{1}{\tau}
$$

The torsion may be positive or negative; if the axis system is a right hand one, the curve, at a point where $\tau>0$, deviates from its osculating circle by bending to the left as one follows $\mathbb{C}$ in the sense of increasing $s$, with head in the direction in which $P$ points.

The geodesic curvature, $x$, is simply related to $R$. The great circle tangent to $\mathbb{C}$ at $P$ has, as unit normal, the vector $P \times T$, and the absolute value of $x$ is the magnitude of the derivative with respect to $s$ of this vector, that is, by (2), $\sqrt{(P \times K)^{2}}$. This reduces, with the help of (3) and (7), to $\sqrt{K^{2}-1}$. In terms of $R$, this is $|\cot R|$. It will be convenient to give $x$ a sign, so we shall identify it with $\cot R^{*}$,

$$
x=\cot R \text {. }
$$

Thus, if a right hand system of axes is postulated, $x>0$ when $\mathbb{C}$ turns toward the left of its tangent great circle.

Combining (14) and (15), we have anew Professor Haskins' result (0, p. 248):

$$
\frac{d}{d s} \tan ^{-1} x=\frac{1}{x}
$$

3. Spherical curves. Osculating and asymptotic circles. For the purposes of applications to problems in dynamics, where coördinates are, in general, analytic functions of the time, and of arc length of paths, it will be adequate to consider curves whose curvatures are, in given intervals, either always increasing, or always decreasing functions of $s$. Accordingly we shall assume in this section, unless the contrary is stated, that in the open interval considered, $s_{0}<s<s_{1}, K^{\prime}$ is either always positive, or else always negative. The fact that $K$ is never negative permits the inequality $\boldsymbol{K}^{\prime}>0$ to embrace two symmetric types of curves between which it is

* See 0, p. 248. The $a$ on the page referred to is the spherical radius of curvature of the intersection of the cone with the sphere. 
desirable to distinguish. We have, indeed, $\varrho^{\prime}<0$, but the first equation (12) shows that $\cos R R^{\prime}<0$, so that $R^{\prime} \gtrless 0$ according as $R \gtrless \pi / 2$. A similar situation obtains with respect to $K^{\prime}<0$. Greater clarity will be attained when it is possible to think of the spherical center of curvature as the nearer of the two points which may play the rôle, so that in case $R>\pi / 2$ for the arc $s_{0}<s<s_{1}$, of $\mathbb{E}$, we shall replace $\mathbb{C}$ by the curve symmetric to it with respect to some diametral plane. The vectors $P, T$ and $K$ will thus go over into symmetrically placed vectors, but $B$ will go over into the symmetric vector with sense reversed, so that the sign of $\cos R$ will be changed. It will be noticed that the hypothesis that $K^{\prime}$ is always positive, or else always negative, on an arc precludes the possibility of an osculating great circle at an interior point of the arc.

The third Frenet formula (13) becomes, with the help of (14),

$$
B^{\prime}=R^{\prime} N
$$

Accordingly, if $\sigma$ represents the arc length of $\mathfrak{F}$, increasing with increasing $s$, the equality of magnitudes and directions in this vector equation yields the result, for the case $R^{\prime}<0$ :

THEOREM III. The tangent to the spherical evolute $\&$ at $Q$ has, for direction, the initial direction of the shorter great circle arc from $Q$ to $P$, and the differential of arc of 5 is given by

$$
d \sigma=-d R \text {. }
$$

Thus, if a flexible inextensible string be unwound from a metal guide having the form of the curve $(5$, the string being kept taut and in contact with the sphere, one of the points of the string will trace the curve $\mathfrak{C}$, so that in this sense the name evolute for $\&$ is appropriate.

The following property of the evolute will also prove useful.

THEOREM IV. No arc of $\&$ on which $R^{\prime}$ is always positive, or always negative, is an arc of a great circle.

Suppose, contrary to the theorem, that $\&$, for $s^{\prime}<s<s^{\prime \prime}$, is an arc of a great circle. Then, if $A$ denote a constant vector perpendicular to the plane of this arc, we have $A \cdot B=0$. If this equation be differentiated, the result reduces, by (16), to $A \cdot N=0$, since $R^{\prime} \neq 0$. But $A \cdot N=0$ and $A \cdot B=0$ imply that $T$ is parallel with $A$, a constant vector, which is impossible for a spherical curve.

We may now infer some properties of a given spherical curve, $\mathfrak{E}$. The first is 
THEOREM V. If $\mathbb{C}$ is an arc of ever increasing curvature, $K^{\prime}>0, s^{\prime}<s<s^{\prime \prime}$, any osculating circle $C_{\mathbf{2}}\left(s=s_{\mathbf{2}}\right)$ lies entirely within any osculating circle $C_{1}\left(s=s_{1}\right)$ for which $s^{\prime} \leqq s_{1}<s_{2} \leqq s^{\prime \prime}$. Here "within" means in that one of the two open regions, into which the circle divides the spherical surface, with the less area.

The theorem is an immediate consequence of equation (17), which, integrated from $s_{1}$ to $s_{2}$, yields $\sigma_{12}=R_{1}-R_{2}$, where $\sigma_{12}$ is the total length of \& between the points characterized by $s_{1}$ and $s_{2}$. Here the vital significance of the hypothesis of monotonic change in $R$ (or $K$ ) appears, for $\sigma$ is always changing in the same sense. Without the hypothesis, \& would have cusps, and $\sigma_{19}$, instead of representing the total length of the arc $\xi_{12}$, would give the algebraic sum of lengths between cusps.

Now $R_{1}$ and $R_{2}$ are less than $\pi / 2$, and, therefore, so is $\sigma_{18}$. The latter, not being the measure of a great circle arc, by Theorem IV, must be greater than $c_{18}$, the length of the geodesic joining the ends of the arc $\mathfrak{E}_{18}$. Hence $c_{12}<R_{1}-R_{2}$. But $R_{1}$ and $R_{8}$ are the spherical radii of the osculating circles $C_{1}$ and $C_{2}$, and $c_{12}$ is the spherical distance between their centers, so that the inequality just derived is the necessary and sufficient condition that $C_{2}$ lie entirely within $C_{1}$.

A more general, though less intuitive, statement may be made with a different sense of "within" and the use of the geodesic curvature, so that osculating great circles are admitted. The curve 5 must cross each of its osculating circles if the geodesic curvature has a derivative different from zero at the point of osculation. If "within" means in that region into which \& enters with increasing $s$, we may state the theorem: if for $s^{\prime}<s<s^{\prime \prime}, x^{\prime}$ is either always positive or always negative, the later osculating circles always lie within the earlier ones. It will be seen how this follows from the theorem as first stated when it is observed that the arc consists at most of two pieces, on one nf which $K^{\prime}>0$, and on the other of which $K^{\prime}<0$.

An immediate corollary of Theorem $\mathrm{V}$ is

THEOREM VI. Under the hypothesis of Theorem V, the arc $s_{1}<s<s_{2}$ of (C) lies entirely within $C_{1}$ and entirely without $C_{8}$. More generally, an arc of $\mathbb{6}$ on which $x^{\prime}$ is always positive, or always negative, lies to one side of its osculating circle at either extremity of the arc.

For if $\mathbb{C}$ cut an osculating circle, or touched it, at a point other than the point of osculation, there would exist two osculating circles with a common point. This is contrary to Theorem V.

One more step yields the analogue for spherical curves of Theorem I:

THEOREM VII. If (5) be supposed to be infinitely long, and if, from some point on, $K^{\prime}>0$, $\mathbb{C}$ approaches an asymptotic circle or point, according as $K$ is bounded or not. If $K^{\prime}<0$, $\mathfrak{c}$ approaches an asymptotic circle. 
We may assume $R<\pi / 2$, as indicated previously. Then as $s$ increases indefinitely, $R$, whose sine is $1 / K$, and which therefore changes monotonely, must approach a limit, $a, 0 \leqq a \leqq \pi / 2$. Accordingly, by (17), $\sigma$ approaches a limit. Hence the center of spherical curvature, $Q$, of $\mathbb{E}$ approaches a limiting position, and as $R$ approaches $a$, it follows that $\mathbb{E}$ approaches the asymptotic circle which has for center the limiting position of $Q$, and for radius, $a$. This circle may reduce to a point if $K$ is increasing, or to a great circle if $K$ is decreasing.

We close this section with the establishment of one more fact, which we shall need in what follows.

THEOREM VIII. If the projections, $x, y, z$, of $P$ have four continuous derivatives with respect to $s$, \& bends, with increasing $s$, to the same or the opposite side of its tangent great circle as $\mathbb{\mathfrak { C }}$, according as $R$ is less than or greater than $\pi / 2$.

To see this, we compare the triple products $\left(B, B^{\prime}, B^{\prime \prime}\right)$ and $\left(P, P^{\prime}, P^{\prime \prime}\right)$, by expressing them in terms of the orthogonal set, $T, N, B$. We start by differentiating the third Frenet formula, $B^{\prime}=-N / \tau$, and simplifying by means of the second:

$$
B^{\prime \prime}=\frac{N \tau^{\prime}}{\tau^{2}}-\frac{N^{\prime}}{\tau}=\frac{N \tau^{\prime}}{\tau^{2}}-\frac{B}{\tau^{2}}+\frac{T}{\varrho \tau},
$$

so that

$$
\left(B, B^{\prime}, B^{\prime \prime}\right)=\left(B,-\frac{N}{\tau}, \frac{T}{\varrho \tau}\right)=\frac{1}{\varrho \tau^{2}}(T, N, B)=\frac{1}{\varrho \tau^{2}} .
$$

On the other hand, $\left(P, P^{\prime}, P^{\prime \prime}\right)=(P, T, K)=(P, T, N) / \varrho$, by (1), (2), and (10). But $P$ must be expressed in terms of $T, N$, and $B: P=(P \cdot T) T$ $+(P \cdot N) N+(P \cdot B) B$, or, by (5), (10), (7) and (12),

$$
P=-\varrho N+\cos R B
$$

Hence $\left(P, P^{\prime}, P^{\prime \prime}\right)=\cos R / \varrho$, and we have, finally, $\tau^{2} \cos R\left(B, B^{\prime}, B^{\prime \prime}\right)$ $=\left(P, P^{\prime}, P^{\prime \prime}\right)$, an equation of which the theorem is a qualitative translation into words. It will be noticed that the hypothesis of differentiability precludes the vanishing of the denominators $\varrho$ and $\tau$ in the above reasoning.

4. Curves with monotonic co-latitude. In a number of dynamical problems connected with the sphere, the motion is limited by two parallel circles. This section will be devoted to a curve, $\mathbb{E}$, on the sphere, bounded by two such circles, the distance of $\mathbb{5}$ from the plane of one of the circles being 
ever increasing, or ever decreasing, and its spherical radius of curvature having the same property. We shall suppose that this curve passes from tangency to $C_{0}$ at $P_{0}$ for $s=s_{0}$ to tangency to $C_{1}$ at $P_{1}$ for $s=s_{1}\left(s_{1}>s_{0}\right)$. The subscripts 0 and 1 will be used generally to distinguish quantities or points connected with the beginning and end, respectively, of this arc of $\mathfrak{c}$. The direction of the common axis of $C_{0}^{v}$ and $C_{1}$, with the sense from the plane of $C_{0}$ to that of $C_{1}$, we shall call north, and take it for that of the $z$-axis. The $x$-axis of our orthogonal axis-system we take in the prime meridian, through $P_{0}$. The $y$-axis we take so as to form with the others a right hand system, or eastward as seen by an observer at $P_{0}$. For the sake of definiteness we shall suppose that $\mathbb{}$ runs initially eastward, a restriction to be removed later.

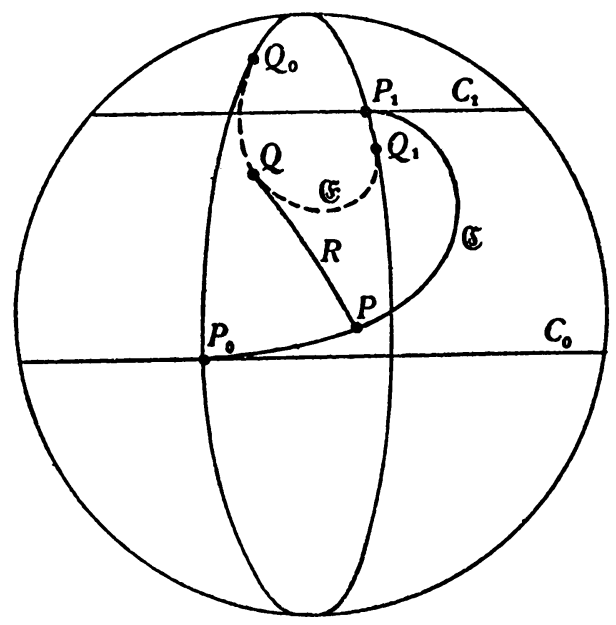

Fig. 1.

THEOREM IX. Let $\mathbb{C}$ be a curve on the unit sphere, $S$, whose position vector, $P$, has four continuous derivatives with respect to $s$ on the closed interval $s_{0} \leqq s \leqq s_{1}$, and let $C_{0}$ and $C_{1}$ denote two parallel circles on $S$, neither of them point circles. Let $\mathbb{C}$ pass from tangency to $C_{0}$ to tangency to $C_{1}$ running initially eastward, under the following conditions on the open interval $s_{0}<s<s_{1}$ : (a) the point $P$, of $\mathbb{C}$, corresponding to the arc-length $s$ has ever increasing distance from the plane of $C_{0}: z^{\prime}>0$,

(b) the spherical radius of curvature, $R$, of $\mathbb{C}$, is ever decreasing: $R^{\prime}<0$.

Then the longitude of $P_{1}$, measured positively to the east, exceeds the longitude of $P_{0}$.

It will be noticed that the hypothesis does not eliminate the possibility of an osculating great circle, for $R$ may exceed $\pi / 2$. It is for this reason that the spherical radius of curvature is more appropriate for the present purpose 
than the curvature, $K$. The particular evolute here used is that defined previously (p. 506).

An intuitive notion of the proof of the theorem may be gained from Figure 1. We compare the longitude, $\psi$, of $P$, on $\Subset$, with the longitude, $\varphi$, of $Q$, on $(5$. Initially $\psi=\varphi=0$, but $\psi$ increases initially more rapidly than $\varphi$, and remains greater, while $\varphi$ is always increasing. Hence $\psi$ is positive at $P_{1}$. What follows is merely an examination of the details.

We begin with an analytic formulation of the hypotheses:

$$
\begin{aligned}
& \text { for } s=s_{0}, s=s_{1}, T \cdot k=0, \\
& \begin{array}{l}
\text { for } s=s_{0}, \\
\text { for } s_{0}<s<s_{1}, \\
\qquad R^{\prime}<0, \\
-1<z_{0}<P \cdot k<z_{1}<+1, \\
(P \cdot k)^{\prime}=T \cdot k>0 .
\end{array}
\end{aligned}
$$

The identity connecting the direction cosines of $k$ with respect to the orthogonal set $T, N, B$ will also prove useful:

$$
(T \cdot k)^{2}+(N \cdot k)^{2}+(B \cdot k)^{2}=1 .
$$

Finally, it will be convenient to employ two vectors in the equatorial plane with the same longitudes as $P$ and $Q$,

$$
p=\frac{P-(P \cdot k) k}{\sqrt{1-(P \cdot k)^{2}}}, \quad b=\frac{B-(B \cdot k) k}{\sqrt{1-(B \cdot k)^{2}}}
$$

in which the denominators do not vanish for $s_{0}<s<s_{1}$ because of the relations $\left(19_{4}\right)$, and $\left(19_{6}\right)$, respectively, with $(20)$.

Our first task is to see that the initial longitudes of $P$ and $Q$ may be taken as 0 . The first, $\psi_{0}=0$, is a matter of definition, and is implied in the term "prime meridian". As $Q$ is always in the plane through $P$ normal to $\mathfrak{C}, Q_{0}$ is in the same meridian plane as $P_{0}$, and, by the definition of $\&$, lies to the north of $P_{0}$. But the important point is that $Q_{0}$ is not separated from $P_{0}$ by the pole (the north pole is meant here and in what follows) on the shorter meridian arc connecting these points. This is because the osculating circle, $C^{\prime}$, of $\mathbb{E}$ at $P_{0}$ cannot go south of $C_{0}$ without carrying $\mathbb{C}$ with it, which is 
contrary to hypothesis $\left(19_{4}\right)$. Hence $Q_{0}$ is either at the pole, or on the prime meridian. In the latter case, its longitude is an integral multiple of $2 \pi$, which may be taken as 0 . If $Q_{0}$ is at the pole, i. e. if $C^{\prime}$ coincides with $C_{0}$, we shall define $\varphi_{0}$ as the limit of $\varphi$ as $s \rightarrow s_{0}$. It will presently appear that this limit exists, and is 0 .

We next show that $\varphi$ is always increasing. To do this, we recall that the magnitude of the derivative of a unit vector is the rate at which it is changing direction, i. e. that $\left|\varphi^{\prime}\right|=\sqrt{\left(b^{\prime}\right)^{2}}$. A little reckoning is required to compute this quantity, but it is straight-forward, and need not be set down. One starts with $\left(21_{2}\right)$ and uses the relations (1), (16) and (20), obtaining

$$
\varphi^{\prime}=\frac{-R^{\prime}(T \cdot k)}{1-(B \cdot k)^{2}}
$$

in which the sign is determined as follows. Because of $\left(19_{1}\right)$ and $\left(19_{3}\right)$, $\varphi^{\prime}$ never vanishes for $s_{0}<s<s_{1}$, and so, being continuous, keeps its sign. By Theorem VIII, $\mathbb{F}$ bends in the same sense as $\mathbb{E}$, or in the sense opposite to that of $\mathbb{E}$, according as $R \gtrless \pi / 2$; hence $\mathbb{E}$ bends to the left. Moreover, by (16), (5) runs initially southward, being tangent to the prime meridian, so that it bends to the east, and $\varphi$ is initially increasing. The equation (22) then shows that $\varphi^{\prime}$ is always positive, and $\varphi$ always increasing, as stated. The same considerations show that $\varphi$ approaches 0 as $s \rightarrow s_{0}$, even if $Q_{0}$ is the pole.

It remains to show that $\psi-\varphi>0$. To do this, we note that by the definition of vector product, $b \times p=k \sin (\psi-\varphi)$. Hence sin $(\psi-\varphi)$ $=(b, p, k)$. Another brief reckoning, involving (20), (18), and the fact that $B \times N=-T$, gives the result

$$
\sin (\psi-\varphi)=\frac{\rho(T \cdot k)}{\sqrt{1-(P \cdot k)^{2}} \sqrt{1-(B \cdot k)^{2}}}
$$

This shows that the angle $\psi-\varphi$, which is continuous, and which starts at 0 , has a positive sine until $P_{1}$ is reached, so that $\psi_{1} \geqq \varphi_{1}>0$, as was to be proved.

More, however, may be inferred from the above developments. The formula (23) shows that as $s \rightarrow s_{1}, \psi-\varphi \rightarrow 0$ or $\pi$. Now, since $R<\pi$, the latter case can occur only when the terminal spherical radius of curvature, which must lie along a meridian, lies across, or terminates in, the pole. This means that the final osculating circle, $C^{\prime \prime}$, contains $C_{1}$ (within that region in which $Q_{0}$ lies), or coincides with $C_{1}$. Also, $\psi-\varphi$ increases toward $\pi$, and $\varphi$ 
is always increasing, so that $\psi$ is increasing at $P_{1}$. The result is that for this case $\mathbb{C}$ touches $C_{1}$ still running eastward. If $\psi-\varphi \rightarrow 0$, as $s \rightarrow s_{1}$, the terminal radius of curvature cannot lie across the pole, and $C^{\prime \prime}$, with spherical radius less than the polar arc to $P_{1}$, must lie south of $C_{1}$, and $C$ then touches $C_{1}$ running west. We accordingly infer

THEOREM X. If to the hypotheses of Theorem IX, we add (c) C touches both circles $C_{0}$ and $C_{1}$ running eastward, then the difference in longitude of its points of contact with these circles exceeds $\pi$.

It remains to consider the case $R^{\prime}>0$ (see Figure 2). As before, we may take $\psi_{0}=0$, and $Q_{0}$ is certainly between $P_{0}$ and the pole. For the former argument

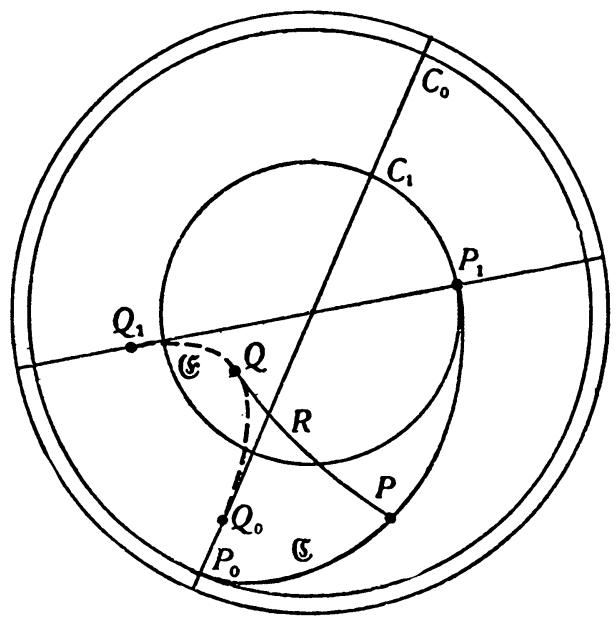

Fig. 2.

shows that $Q_{0}$ is not beyond the pole; and no more can $Q_{0}$ coincide with the pole, for from (16) we infer that $-\operatorname{cosec}^{8} R R^{\prime}=x^{\prime}$, so that $x^{\prime}$ would be negative, i. e. $x$ decreasing, and $\mathbb{E}$ would have to bend to the south of $C_{0}$, contrary to hypothesis. As to $\mathfrak{F},(16)$ now shows that it runs northward, and hence to the west, by Theorem VIII. As $R^{\prime}>0$, formula (22) is valid without change, and $\varphi$, initially 0 , continually decreases. But by $(23), \sin (\psi-\varphi)$ is positive, and it approaches 0 as $s \rightarrow s_{1}$.

If $\psi-\varphi \rightarrow 0$, the terminal spherical radius of curvature cannot cross the pole, su that the spherical radius of $C^{\prime \prime}$ is less than that of $C_{1}$. Hence, as $C^{\prime \prime}$ cannot lie north of $C_{1}$, its center also lies south of $C_{1}$. As $\varphi$ is decreasing, and $\psi-\varphi$ approaching 0 through positive values, we infer that $\psi$ is decreasing, and that in this case $\mathbb{C}$ touches $C_{1}$ running west. We have here, $\psi_{1} \leqq \boldsymbol{g}_{1}<0$, and the result is merely Theorem IX with the rôles of $C_{0}$ and $C_{1}$ interchanged, and $\mathbb{C}$ replaced by its reflection in the prime meridian. 
But if $\psi-\varphi \rightarrow \pi$, the terminal radius of curvature lies across the pole, and we infer that $\sqrt{ }$ is still running eastward at $P_{1}$, so that we may state

THEOREM XI. If in Theorem IX we replace the condition (b) by $\left(b^{\prime}\right)$ the spherical radius of curvature of $\mathbb{C}$ is always decreasing, $R^{\prime}<0$, and add the condition $(c)$ of Theorem $\mathrm{X}$, it then follows that the difference in longitude of the points of contact of $\mathbb{E}$ with the circles $C_{0}$ and $C_{1}$ is less than $\pi$.

Finally, we consider the removal of the restriction that $\mathbb{S}$ run initially eastward. If $\mathbb{c}$ runs initially westward, we may consider its reflection in the plane of the prime meridian. The proofs of the corresponding theorems remain the same, except that the other evolute from the one we have been employing must be used. Or, we may use a left hand system of axes, which will produce the same effect. The three last theorems may now be stated as follows.

Let (5) run from tangency at $P_{0}$ to $C_{0}$, to tangency at $P_{1}$ to $C_{1}$, the distance of the moving point, $P$, on $\mathbb{E}$, from the plane of $C_{0}$ having a positive derivative with respect to the arc length $s$ for $s_{0}<s<s_{1}$. Let $R$ be the spherical radius of curvature of $\mathbb{C}$ which is measured initially toward $P_{0}$ from the side of $C_{0}$ on which $C_{1}$ lies. Then

(1) if $R^{\prime}<0$, or if $R^{\prime}>0$ for $s_{0}<s<s_{1}$, that one of the points $P_{0}, P_{1}$, for which $R$ is the less, has the greater longitude, measured_in the sense of increasing arc length at $P_{0}$;

(2) if $R^{\prime}<0$ for $s_{0}<s<s_{1}$, and the longitude of $P$ is changing in the same sense at $P_{0}$ and at $P_{1}$, the difference in longitude of these two points exceeds $\pi$;

(3) if $R^{\prime}>0$ for $s_{0}<s<s_{1}$, and the longitude of $P$ is changing in the same sense at $P_{0}$ and at $P_{1}$, the difference in longitude of these two points is numerically less than $\pi$.

In the above statements, the geodesic curvature, $x$, might have been used instead of $R$, but it would have necessitated a subdivision of cases according as $x \gtrless 0$, or else a modification of the convention as to the sign of $x$. Neither seems desirable.

\section{PART II. SOME POINTS IN THE THEORY OF THE TOP}

5. Osgood's intrinsic equations. Notation. Some familiarity on the part of the reader with Professor Osgood's paper will be assumed in what follows. His intrinsic equations, however, will appear here with a change in a sign, and a slight change in notation.* We write them as follows:

\footnotetext{
* The change in sign is due to a different convention as to the senses in which certain quantities are measured. In justification of a departure from Professor 0sgood's well considered conventions, I can only plead that I have felt surer footed in employing conventions to which I have been accustomed. Since there is little agreement in the literature on the subject, the departure will cause the reader little inconvenience.
} 


$$
\begin{aligned}
4 v \frac{d v}{d s} & =T, \\
A v^{2} x-C r v & =N, \\
C \frac{d r}{d s} & =S .
\end{aligned}
$$

Here $C$ and $A$ are the moments of inertia of the top about its axis of dynamic symmetry, and a perpendicular to the axis through the fixed point, respectively. The magnitude of the velocity of the point $P$, where the axis of the top (on which a positive sense has been arbitrarily fixed) pierces the unit sphere, is denoted by $v$; the geodesic curvature of $\mathfrak{E}$, the path of $P$, or the "bending" of the cone swept out by the axis of the top, is denoted by $x ; x$ is positive when $\mathbb{C}$ swerves to the left of an observer walking on the sphere and following the top axis. The sense of the positive unit tangent vector, $t$, to $\mathbb{E}$, is that of the motion, and that of the normal vector, $n$ (here tangent to the sphere, and not to be confused with the principal normal vector, $N$, of Part I), is to the left of the above mentioned observer. Thus a curve with positive $x$ bends toward the positive normal. The component of the applied moment, along the axis, or the spin moment, is denoted by $S$. The remaining component of the applied moment may be regarded as due to a force tangent to the sphere and applied at $P$. We denote the components of this force in the directions of $t$ and $n$ by $T$ and $N$, respectively. In Professor Osgood's notation, this force has components $T$ and $Q$ along the tangent and negative normal vectors associated with c, but his normal vector points to the right, so that his $Q$ and the present $N$ are identical. Here, a positive moment or rotation about an axis would force a right hand screw forward in the positive sense along the axis. Thus our $S$ and $r$ are opposite in sign to the corresponding $N$ and $r$ of Professor Osgood' - paper. The only change in sign resulting from these differences is in that of the term $\mathrm{Crv}$ in the second intrinsic equation.

6. The energy integral. This is obtained, in case it exists, from equations $\left(24_{1}\right)$ and $\left(24_{3}\right)$, and may be written

$$
\frac{1}{2}\left(A v^{2}+C r^{2}\right)=\int\left(T+S v \frac{d r}{d s}\right) d s+h,
$$

provided the integrand is the derivative with respect to $s$ of a function of position, as it is, for instance, in the case of the heavy frictionless top with fixed peg $(0$, p. 256 , (i)). 
7. The heavy top with fixed peg. Initial sense of change of latitude. The equations of the motion may be written $(0$, p. $258,(6)$ and (iv), p. 256, (i))

$$
\begin{aligned}
\left(\frac{d u}{d t}\right)^{2} & =P(u), \\
\frac{d \psi^{\prime}}{d s} & =\frac{\varepsilon+\gamma\left(u_{0}-u\right)}{v\left(1-u^{2}\right)}, \\
\varkappa & =\frac{\gamma}{v^{3}}\left\{v_{0}^{2}-\frac{a \varepsilon}{2 \gamma}+\frac{a}{2}\left(u_{0}-u\right)\right\}, \\
v^{2} & =v_{0}^{2}+a\left(u_{0}-u\right) .
\end{aligned}
$$

Here $u=\cos \theta, \theta$ being the co-latitude of $P ; \psi$ is the longitude of $P$, positive when measured eastward; $u_{0}, \psi_{0}, \dot{\psi}_{0}$, and $v_{0}$, are initial values, corresponding to some moment when $\dot{\theta}(=d \theta / d t)=0 ; \varepsilon=\left(1-u_{0}^{2}\right) \dot{\psi}_{0}$; $v_{0}^{2}=\sin ^{2} \theta_{0} \dot{\psi}_{0}^{2}=\left(1-u_{0}^{2}\right) \dot{\psi}_{0}^{2} ; \nu$ is the constant value of $r ; \gamma=C \nu / A ; *$ $a=2 M g h / A$, where $M$ is the mass, and $h$ the distance of the center of mass from the fixed point. The function $P(u)$ is given by

$$
P(u)=\left(1-u^{2}\right)\left[v_{0}^{2}+a\left(u_{0}-u\right)\right]-\left[\varepsilon+\gamma\left(u_{0}-u\right)\right]^{2} .
$$

The motion takes place between two parallel circles, $C_{0}$ and $C_{1}$, which may, in special cases, reduce, one or both, to point circles. We shall disregard such special cases in the present study.

The first question that arises when the initial conditions include $\theta_{0}=0$, as at present, is, does the top begin to rise, or to fall, or, is the initial limiting circle the lower, $C_{0}$, or the upper, $C_{1}$ ? To answer this, one compares the initial value of $x$, or

$$
x_{0}=\frac{1-u_{0}^{2}}{v_{0}^{8}}\left[\gamma \dot{\psi}_{0}^{2}-\frac{a}{2} \dot{\psi}_{0}\right]
$$

* We shall assume $\nu$, and hence $\gamma$, to be positive, or when so specified, zero; never negative. It will be recognized that this restriction merely involves, in some cases, reflecting the motion in a mirror. 
with the geodesic curvature, $x_{c}$, of the initial limiting circle. But $\varkappa_{c}=\operatorname{sgn} \dot{\psi}_{0} \cot \theta=\operatorname{sgn} \dot{\psi}_{0} \frac{u_{0}}{\sqrt{1-u_{0}^{2}}}$, or, as $v_{0}=\sqrt{1-u_{0}^{2}} \operatorname{sgn} \dot{\psi}_{0} \dot{\psi}_{0}$, $\varkappa_{c} v_{0}^{3}=u_{0}\left(1-u_{0}^{2}\right) \dot{\psi}_{0}^{3} *$ Hence

$$
\left(x_{0}-x_{c}\right) \dot{\psi}_{0}=-\frac{1}{v_{0}}\left[u_{0} \dot{\psi}_{0}^{2}-\gamma \dot{\psi}_{0}+\frac{a}{2}\right] \text {. }
$$

If $x_{0}>x_{c}, \mathbb{E}$ bends more rapidly to the left than the initial limiting circle, i. e. if running east, $\mathbb{5}$ rises, and similarly for the other cases, the sign of $\left(x_{0}-x_{c}\right) \dot{\psi}_{0}$ being decisive. The function in brackets is the quadratic whose roots give the longitudinal velocities of steady precession in the circle of latitude $\theta=\theta_{0}$. If $u_{0}>0,\left(x_{0}-x_{c}\right) \dot{\psi}_{0}$ is positive between the roots of the quadratic, and if $u_{0}<0$, the opposite is the case. If $u_{0}=0$, there is but one root, and $\left(x_{0}-x_{c}\right) \dot{\psi}_{0}>0$ for $\dot{\psi}_{0}$ greater than this root. Hence we may state

THEOREM XII. If, at any instant, the path of $P$ touches a circle of latitude, it will curve away from, or toward, the equator according as its longitudinal velocity at the instant does, or does not, lie between the longitudinal velocities for steady precession in that circle. If the circle is the equator, the path curves northward or southward according as the longitudinal velocity does, or does not exceed the single longitudinal velocity for steady precession on the equator. If the spin is so slight that the precessional values are coincident, or imaginary, the top always falls.

It is, of course, understood that the initial longitudinal velocity is not a precessional value in the statement of the above theorem.

8. Reversals in sense of the longitudinal motion. Equation $\left(26_{2}\right)$ gives the value of $u$ for which the longitudinal motion changes sense: $u_{l}=u_{0}$ $+\varepsilon / \gamma$. In order, however, that this value of $u$ shall correspond to a real point in the motion, it is necessary first, that it lie in the closed interval $(-1,+1)$, and secondly, that $P\left(u_{l}\right) \geqq 0$. As $P(u)<0$ if $u<-1$, these conditions may be stated as follows:

$$
\dot{\psi}_{0} \leqq \frac{\gamma}{1+u_{0}}
$$

$$
P\left(u_{l}\right)=\frac{\left(1-u_{0}^{2}\right)^{3}}{\gamma^{2}} \dot{\psi}_{0}\left(\dot{\psi}_{0}+\frac{\gamma}{1+u_{0}}\right)\left(\frac{\gamma}{1-u_{0}}-\dot{\psi}_{0}\right)\left(\dot{\psi}_{0}-2 \delta\right) \geqq 0,
$$

* The function $\operatorname{sgn} z$ is the familiar function signum $z$, defined as follows: for $z<0$, $\operatorname{sgn} z=-1$, for $z=0, \operatorname{sgn} z=0$, and for $z>0, \operatorname{sgn} z=+1$. 
where $\delta=a / 2 \gamma$ is the value of the longitudinal velocity for steady precession on the equator.

9. Points of inflection. The curve $\mathbb{S}$ has a point of inflection at $P$ when its projection on the plane tangent to the sphere at $P$ has a point of inflection at $P$, or, when $(C$ crosses an osculating great circle. A necessary condition for this is $x=0$; it is sufficient that $x$ change signs. Equation $\left(26_{3}\right)$ gives, as the only value of $u$ for which this can occur, $u_{i}==u_{0}+\left(2 v_{0}^{2} / a\right)-(\varepsilon / \gamma)$. Here, the conditions that $u_{i}$ correspond to a real point in the motion may be given the form

$$
\begin{aligned}
& F_{1}\left(\dot{\psi}_{0}\right)=\dot{\psi}_{0}^{2}-\delta \dot{\psi}_{0}-\frac{u}{2\left(1+u_{0}\right)} \leqq 0 \\
& \begin{aligned}
P\left(u_{i}\right)=\frac{4\left(1-u_{0}^{2}\right)^{3}}{a^{2}} & \dot{\psi}_{0}\left(\dot{\psi}_{0}-\delta\right)\left(\dot{\psi}_{0}-2 \delta\right) \\
& \times\left[\left(\dot{\psi}_{0}-\delta\right)\left(\dot{\psi}_{0}^{2}-\frac{\gamma^{2}}{1-u_{0}^{2}}\right)+\frac{a u_{0}}{1-u_{0}^{2}} \dot{\psi}_{0}\right] \geqq 0 .
\end{aligned}
\end{aligned}
$$

The factorization in the value of $P\left(u_{i}\right)$ has been carried as far as possible in the domain of rationality $\left(a, \gamma, u_{0}, \sqrt{1-u_{0}^{2}}\right)$. This may be verified by considering the special rational values $a=16 / 5, \gamma=4 / 5, \delta=2, u_{0}=3 / 5$, which reduce the cubic factor to the form $x^{3}-2 x^{2}+2 x+2$, a polynomial evidently without rational roots.

10. Monotonic curvature. For the application of some theorems of Part I, we need assurance that $\boldsymbol{x}^{\prime}$ keeps its sign between the limiting circles. This derivative, obtained from (26, may be reduced, without excessive trouble, to

$$
\varkappa^{\prime}=\frac{a \gamma}{v^{5}} u^{\prime}\left[v_{0}^{2}-\frac{3 a \varepsilon}{4 \gamma}+\frac{a}{4}\left(u_{0}-u\right)\right]
$$

Striking is the fact that like the curvature, $x$, and the longitudinal velocity, $\dot{\psi}$, this derivative is dependent for its sign on a linear function of $u$. The derivative, $u^{\prime}$, of $u$, keeps its sign between the limiting circles. The value of $u$ for which $x^{\prime}$ vanishes is $u_{k}=u_{0}-(3 \varepsilon / \gamma)+\left(4 v_{0}^{2} / a\right)$, and the conditions that it characterize a real point in the motion may be given the form 


$$
\begin{aligned}
& F_{2}\left(\dot{\psi}_{0}\right)=\dot{\psi}_{0}^{2}-\frac{3 \delta}{2} \dot{\psi}_{0}-\frac{a}{4\left(1+u_{0}\right)} \leqq 0, \\
& P\left(u_{k}\right)=\frac{48}{a^{2}}\left(1-u_{0}^{2}\right)^{3} \dot{\psi}_{0}\left(\dot{\psi}_{0}-\frac{3}{2} \delta\right)\left(\dot{\psi}_{0}-2 \delta\right) \\
& \quad \times\left[\dot{\psi}_{0}^{2}\left(\dot{\psi}_{0}-\frac{3}{2} \delta\right)-\frac{a\left(a-6 \delta^{2} u_{0}\right)}{12 \delta^{2}\left(1-u_{0}^{2}\right)}\left(\dot{\psi}_{0}-\frac{a \delta}{2\left(a-6 \delta^{2} u_{0}\right)}\right)\right] \geqq 0 .
\end{aligned}
$$

The cubic factor is, in this case also, irreducible in the domain $\left(a, \gamma, u_{0}\right.$, $\left.\sqrt{1-u_{0}^{2}}\right)$, as may be seen by using the special values $a=24, \delta=2, u_{0}=0$.

11. Applications of the geometry of spherical curves. It is not the purpose of the present paper to enter into a detailed discussion of the various cases of motion of the top which may present themselves, although the materials gathered above permit new distinctions between types.* We shall rather content ourselves with the enunciation of certain typical results, following the customary cases $(0$, p. 258). $t$

Case I. The longitude is always increasing $(0$, Figure $3, I)$. In this case, it is usually assumed that the path of $P$ has points of inflection. The illustrations of the most current text books show such a curve. $\neq$ It should be noticed, however that such is not necessarily the case, and that in the present type of motion both paths with inflections and paths without inflections can occur. Thus, for $a=2, \delta=\gamma=1, u_{0}=1 / 2$, with $\dot{\psi}_{0}$ near to 1 , the condition $\left(30_{1}\right)$ for a change in sense of the longitudinal motion is not fulfilled, so that the longitude is always increasing. The condition $\left(31_{1}\right)$ for a real point of inflection is fulfilled, while the condition $\left(31_{2}\right)$ takes the form $\dot{\psi}_{0}-1 \leqq 0$ for $\dot{\psi}_{0}$ near 1 . Thus, with $\dot{\psi}_{0}$ slightly less than 1 , we have a path with a point of inflection on each arc between the limiting circles, and with $\dot{\psi}_{0}$ slightly greather than 1 , we have an inflectionless path.

If the spin be stopped $(\gamma=0)$, the top becomes a spherical pendulum. The longitude always changes monotonely, $\left(26_{2}\right)$, and $x$ reduces, by $\left(26_{3}\right)$ to $-a \varepsilon / 2 v^{3}$. Thus the path of the spherical pendulum never has inflection points. The use of the intrinsic equations has rendered extremely simple the proof of a well known fact.

*Mr. A. H. Copeland, of the Graduate School at Harvard, is undertaking such a discussion in connection with his candidacy for the doctorate.

† Case I is the wavy curve without cusps or double points, Case II is the curve with cusps, and Case III, the curve with loops.

$\ddagger$ Professor Osgood has also overlooked the necessity of imposing the conditions (31) on $\dot{\psi_{0}}$ in order to secure a path with inflection points (see 0, p. 259). 
Another fact about the spinning top that seems hitherto to have escaped explicit mention is the following: there exist cases in which the longitude of $P$ increases by more than $\pi$ between two successive contacts with the limiting circles.*

Sufficient conditions for this type of motion are (see Theorem $X$ ): the longitude is always increasing, the top is rising from $u=u_{0}$, and $R^{\prime}<0$ (the definitions of $R$ in Section 2 and Section 4 coincide in the present case, and we find from (15) that the last condition is equivalent to $x^{\prime}>0$ ). An example showing the compatibility of these conditions is the following: $a=1, \gamma=1$, $u_{0}=0.4, \dot{\psi}_{0}=1.44312$. We find $u_{1}=0.5, \varepsilon=1.21222, v_{0}^{2}=1.74938$. The condition $\left(30_{1}\right)$ for a change in sense of the longitudinal motion is contradicted, while $\dot{\psi}$ is initially positive; $x^{\prime}$ is seen from (32) to be initially positive, while the value $u_{k}$ (p. 519) for which $x^{\prime}$ changes sign is found to be greater than 1, and so does not correspond to a real point on the path.t

Case II. Here $\dot{\psi}_{0}=0$, and by.(32), $x^{\prime}$ can vanish only for $u=u_{0}$. We infer, from Theorem VI, that an arc of the path of $P$ between two successive contacts with the limiting circles lies entirely within the osculating circle at the extremity of the arc at which the curvature is finite.

Case III. The same situation obtains here, where the path has loops. Let us use $\mathbb{C}$ to denote an arc of the path between the limiting circles. Then $\mathbb{E}$ lies entirely within its osculating circle at one extremity, and entirely without its osculating circle at the other extremity, one of these circles containing the other in its interior, by Theorems V and VI. "Interior" may here be interpreted in the narrower sense, namely, the region with the less area.

To justify these statements with regard to Case III, we must show that for the looped curve, neither $x^{\prime}$ nor $x$ vanishes between the limiting circles, i. e. that the conditions (33) and (31) are both incompatible with the conditions (30), when the strict inequalities are employed in the first two. We shall give the proof for the conditions (33), that for (31) being similar, and simpler.

In the first place, it is no restriction to assume that $\dot{\psi}_{0}>0$, for this merely means that a proper choice has been made of that limiting circle which is to be the initial one, inasmuch as $\sqrt{5}$ meets the limiting circles running in

* If $P(u)$ has $u=1$ as a double root, $\sqrt{ }$ makes a spiral around the north pole. It seems entirely plausible that near this motion are others of the type in question, where the increase in longitude is arbitrarily large. It may also be of interest to note that a similar situation exists in a very elementary problem, namely, the following: a bead is free to slide under gravity without friction on a circular wire, which rotates with constant angular velocity about a vertical diameter. It will be found that the wire makes more than a half revolution between two successive times when the bead attains its extreme heights.

† A computation, which I believe to be accurate, gives, to four significant figures, $\Psi_{01}=3.428$, which is about 9.1 percent in excess of $\pi$. 
opposite senses. This assumption greatly simplifies $\left(30_{2}\right)$, reducing it to $\dot{\psi}_{0}-2 \delta>0$, so that the conditions for the loopy type of curve become

$$
2 \delta<\dot{\psi}_{0} \leqq \frac{\gamma}{1+u_{0}} .
$$

We proceed to show that for $\dot{\psi}_{0}$ thus limited, the conditions derived from (33),

$$
\begin{gathered}
\dot{\psi}_{0}^{2}-\frac{3}{2} \delta \dot{\psi}_{0}-\frac{a}{4\left(1+u_{0}\right)}<0 \\
\dot{\psi}_{0}^{2}\left(\dot{\psi}_{0}-\frac{3}{2} \delta\right)-\frac{a\left(a-6 \delta^{2} u_{0}\right)}{12 \delta^{2}\left(1-u_{0}^{2}\right)} \dot{\psi}_{0}+\frac{a^{2}}{24 \delta\left(1-u_{0}^{2}\right)}>0
\end{gathered}
$$

are incompatible. If the first of these inequalities be multiplied by $\dot{\psi}_{0}$ and subtracted from the second, we find a necessary condition on $\dot{\psi}_{0}$ for their consistency which reduces to $\dot{\psi}_{0}\left[3 \delta^{2}\left(1+u_{0}\right)-a\right]+a \delta / 2>0$. From (34), recalling that $\gamma=a / 2 \delta$, we have $a>4 \delta^{2}\left(1+u_{0}\right)$, so that the coefficient of $\dot{\psi}_{0}$ is negative, and

$$
\dot{\psi}_{0}<\frac{a \delta / 2}{a-3 \delta^{2}\left(1+u_{0}\right)}
$$

If from this we form the inequality for $\dot{\psi}_{0} / 2 \delta$, and in it substitute $4 \delta^{2}\left(1+u_{0}\right)$ $=a(1-x)$, so that $x$ is always positive, we find as upper bound for $\dot{\psi}_{0} / 2 \delta$ a function of $x$ whose maximum is 1 , whereas, by (34), $\dot{\psi}_{0} / 2 \delta$ must be greater than 1. Thus the incompatibility of the conditions (33) is established.

We may state further concerning this case, the consequence of Theorem IX: the general drift of the longitudinal motion is in the sense which it has at that one of the limiting circles where the curvature is numerically the less. This is a property whose proof has previously required less elementary methods (see the reference, 0, p. 260).

If the spin of the top be stopped again, so that the spherical pendulum is before us, it is known that the difference in longitude between two contacts of the path with its limiting circles exceeds $\pi / 2$ and is less than $\pi$. The first inequality is obtained by the appraisal of a definite integral. The second has required the use of Cauchy's integral theorem, or other less elementary method (see Appell, loc. cit.). It is readily verified that this second inequality is an immediate consequence of Theorem XI. The hypothesis of the theorem which 
interests us may be given the form $\dot{\psi}_{0} \kappa^{\prime}<0$, for with the definition of $R$ there employed, the relation (15) is to be replaced by $x=\operatorname{sgn} \dot{\psi}_{0} \cot R$, when we consider a rising arc, $\sqrt{ }$, of the path. For $\gamma=0,(32)$ takes the form $x^{\prime}=-3 a^{2} u^{\prime}\left(1-u_{0}^{2}\right) \dot{\psi}_{0} / 4 v^{5}$, so that the hypothesis is fulfilled.

12. Asymptotic circles. On page 225 of his article, Professor Osgood mentions an interesting case of motion of the symmetric top, in which it is subjected to a force of constant magnitude, directed always along the positive tangent to the path, $\mathfrak{C}$. He says that $x$ now approaches 0 , and "it is a matter of conjecture as to whether $\mathbb{C}$ has an asymptotic great circle". Inasmuch as it appears rather obvious that it should have, the statement might seem to be excessively cautious. But the example given in Section 1 shows such caution entirely justified. As a matter of fact, $\mathbb{5}$ has an asymptotic great circle, by Theorem VII. For, if $f$ denote the magnitude of the force, we find $s=\frac{f}{2 A}\left(t-t_{0}\right)^{2}+v_{0}\left(t-t_{0}\right)$, so that the path is infinitely long; also that $x=C \nu / A v$, where $v=\sqrt{v_{0}^{2}+\frac{2 f}{A} s}$, so that $x>0$ and $x^{\prime}<0$, and hence $K^{\prime}<0$. The hypotheses of Theorem VII are therefore fulfilled.

Certain general criteria may be set up for motion with an asymptotic circle. We shall suppose that from some point $\left(t=t_{0}, s=s_{0}\right)$ on, $v$ does not vanish, and that the functions involved have whatever continuous derivatives are required. Then the path will be of infinite length if the integral

$$
t-t_{0}=\int_{s_{0}}^{s} \frac{d s}{\sqrt{v_{0}^{2}+\frac{2}{A} \int_{s_{0}}^{s} T d s}}
$$

is real and finite for all $s>s_{0}$. This is a first condition.

If we differentiate with respect to $s$ the equation (24, , and simplify the result by means of $\left(24_{1}\right)$ and $\left(24_{3}\right)$, we obtain

$$
A v^{8} \varkappa^{\prime}=\frac{d N}{d s}+S-\frac{T(C r v+2 N)}{A v^{2}} .
$$

That the right hand member of this equation, which we assume to be continuous, should never vanish, is the second of the desired conditions. 
In the case of a purely tangential force, the latter condition takes the following form: $T$ shall not vanish for $s>s_{0}$. If $T$ is negative, the motion comes to a halt unless $\int_{s_{0}}^{s}(-T) d s<A v_{0}^{2} / 2$ for all $s>s_{0}$. Otherwise, the path has an asymptotic circle, which may, however, reduce to a point provided $T<0$ and $v \rightarrow 0$, i.e. $\int_{s_{0}}^{\infty}(-T) d s=A v_{0}^{2} / 2$. The asymptotic circle is a great circle if $T>0$ and $\int_{0}^{s_{0}} T d s$ is divergent.

HARVARD UNIVERSITY, Cambridge, Mass. 